\title{
A Negotiation Protocol for Multiple Interdependent Issues Negotiation Over Energy Exchange
}

\author{
Muddasser Alam, Alex Rogers and Sarvapali D. Ramchurn \\ Intelligence, Agents, Multimedia Group, \\ School of Electronics and Computer Science \\ University of Southampton, UK. \\ $\{$ ma606, acr, sdr\}@ecs.soton.ac.uk
}

\begin{abstract}
We present a novel negotiation protocol to facilitate energy exchange between off-grid homes that are equipped with renewable energy generation and electricity storage. Our solution imposes additional constraints on negotiation such that it reduces a complex interdependent multi-issue problem to one that is tractable. We prove that using our protocol, agents can reach a Pareto-optimal, dominant strategy equilibrium in a decentralized and timely fashion. We empirically evaluate our approach in a realistic setting. In this case, we show that energy exchange can be useful in reducing the capacity of the energy storage devices in homes by close to $40 \%$.
\end{abstract}

\section{Introduction}

It is estimated that 1.6 billion people live without access to electricity; mostly in the Indian subcontinent and SubSaharan Africa [IEA, 2008]. This population is divided in very small communities scattered over vast areas of land and hence, providing them with centralized power distribution has not been justified to date due to the huge capital cost of such infrastructure and the lack of demand. However, the opportunity for demand cannot become established if there is no supply. To address this dilemma, a number of initiatives have begun to provide these communities with off-grid renewable microgeneration. infrastructure such as solar panels, and electric batteries for storage ${ }^{1}$. However, these resources are operated in isolation. Our aim is to investigate whether the interconnection and decentralized coordination of such resources could result in their more efficient use and be beneficial to the connected homes in the community. We envision that such interconnection and coordination could lead to the creation of a grid from the ground up and allow these communities to take an evolutionary jump straight to the smart grid, in the same way that many of them have bypassed landlines and leapt straight to cell phone technology.

As a first step towards this vision, we investigate the possibility of exchanging energy between two interconnected homes. The core challenge is to enable the autonomous coordination of energy infrastructure in these individual households to ensure that energy is used efficiently. Given the lack of a centralised system, absence of banking/payment systems and low-processing power at hand, we need a tractable and decentralized energy exchange solution, that can operate without financial payments between houses, and provides a benefit to all participants, compared to being disconnected.

Now, the idea of energy exchange is not new. There are several real world examples where energy exchange is used to improve energy management between countries (e.g. Finland and Sweden) and between cities (e.g. Dehli and Madya Pardesh, India). Indeed, exchange of energy has already been shown to result in efficient use of energy and cost savings in utility companies [Ehtamo et al., 1988]. In this context, Ruusunen et al. [1991] considered a group of utility companies, each owning a generator, connected together to form a power pool. The cost of energy generation was different for each company and varied over the course of a day, allowing energy exchange to be beneficial to all. In their solution, energy generation and consumption is monitored by the pool operator who audits the cost and utility functions of the participants and distributes the cost savings among them. The energy exchange takes place according to a static exchange agreement which is established manually, prior to the exchange.

In contrast, Rosenschein and Zlotkin [1994] argued that in general, such mediated solutions are unnecessary, and automatic negotiation between software agents is a more efficient approach. They seek to design the rules of interaction for these negotiations such that a society of agents, as a whole, exhibits desirable properties such as stability (e.g. Nash equilibrium), efficiency (Parteo-optimality) and simplicity (strategy-proofness). Agents interact with each other in a decentralized manner obeying the rules of encounter. Careful design of such rules prevents the agents from being able to exploit the system and also reduces the overall complexity of interaction for the agents. They show examples of such rules for certain domains but conclude that there is no universal set of rules for general encounters and, therefore, a specific set of rules is required for a specific domain. Our work is in line with this approach, however, our setting is more challenging than the ones they consider since negotiation over energy exchange is multi-issue because agents are required to decide over the amount of energy exchange and also, how this exchange is scheduled across the day. Furthermore, these issues are interdependent as the recipient's utility for any period may depend on the energy received in earlier periods (since energy can be stored in our setting).

\footnotetext{
${ }^{1}$ See the Rural Solar Homes in India (www.tatabpsolar.com), the Solar Homes program in Bangladesh (www.gshakti.org) and the Solar Village program in Ethopia (www.solarsenegal. com).
} 
More recent work addressing interdependent issues has focused on two tracks. Hindriks et al. [2006] attempt to remove interdependencies by approximating the utility space. However, they conclude that their technique only works with certain classes of utility functions where issues are not highly dependent (which is not the case in the problem of energy exchange) and cannot be applied to more general cases. Hattori et al. [2007], Ito et al. [2007], and Fujita et al. [2010] take a different approach and assume a central authority (a mediator) to which agents provide information about their utility functions. This centre calculates the set of Pareto-optimal solutions, from which the agents choose one. However, these solutions require the presence of an unbiased and independent mediator, capable of carrying out intensive computations. Such assumptions are hard to hold in our decentralized setting where there is no centre and households are required to negotiate directly to each other.

In contrast, in this paper, we address the problem of interdependent multi-issues negotiation to exchange energy, by providing a negotiation protocol which imposes three key restrictions on the offers that agents can make, and effectively reduces this problem to a single-issue negotiation problem but only minimally reduces the efficiency of the solutions found. We show that our approach leads to a Pareto-optimal, dominant strategy equilibrium and makes it easier for agents to compute optimal offers. In more detail, this work advances the state-of-the-art in the following ways:

1. We present a novel negotiation protocol that reduces the complexity of interdependent multi-issue negotiation in energy exchange problem, to a single-issue negotiation.

2. We prove that: (i) this protocol leads to a dominant strategy equilibrium and (ii) the outcome is Pareto-optimal. Offers can be calculated optimally using linear programming and the negotiation stops in two rounds.

3. We empirically evaluate our protocol in an example and show that in this case (i) the energy saved using our protocol can be up to $84 \%$ of the energy that can be saved using the Nash bargaining solution (ii) agents can increase their utility via our protocol up to $22 \%$ compared to utility without exchange and (iii) agents can maintain their utility with less battery storage (up to $37 \%$ less).

The rest of the paper is structured as follows. Our model is explained in Section 2 followed by a discussion of a theoretical solution in Section 3. Section 4 presents our negotiation protocol and its properties. We evaluate our protocol in a specific setting in Section 5 and conclude in Section 6.

\section{Energy Exchange In Homes: the Model}

In this section, we outline a model of energy exchange in two connected homes, each with a renewable generation unit, some loads and a battery to store electricity. Specifically, let $a$ be an agent with a generation capability $\boldsymbol{k}=\left(k_{1}, \ldots, k_{n}\right)$ representing the energy it can generate in $n$ time periods and a load $\boldsymbol{h}=\left(h_{1}, \ldots, h_{n}\right)$ representing its loads requirements. The battery is characterised by four parameters: a maximum storage capacity, $s$, a maximum charging rate, $c_{\max }$, maximum discharging rate, $d_{\max }$, and efficiency $e$. The efficiency describes the loss of energy when the battery is charged. We describe the dynamic state of the battery by: the energy flow into the battery (charge) $c=\left(c_{1}, \ldots, c_{n}\right)$, the flow going out (discharge) $\boldsymbol{d}=\left(d_{1}, \ldots, d_{n}\right)$ and the amount of charge stored in battery at any given time $\boldsymbol{q}=\left(q_{1}, \ldots, q_{n}\right)$.

The generation capability $\boldsymbol{k}$ denotes the energy that can be generated, however, an agent may reduce its generation if the energy to be generated can neither be used immediately nor stored due to the limited battery flow or capacity. To capture this possibility, we use a generation, $\boldsymbol{g}=\left(g_{1}, \ldots, g_{n}\right)$, to indicate the actual energy generated and wasted energy, $\boldsymbol{w}$, to denote the energy that was not generated or wasted. It is obvious that $\boldsymbol{k}=\boldsymbol{g}+\boldsymbol{w}$.

Using the battery, an agent can compute an energy allocation, $\boldsymbol{p}=\left(p_{1}, \ldots, p_{n}\right)$, allocating the generated energy $\boldsymbol{g}$ to loads $\boldsymbol{h}$. Finally, the link flow $\boldsymbol{l}=\left(l_{1}, \ldots, l_{n}\right)$ is used to denote the flow on the link between agents.

The utility of agent $a$ at time $t$ is the ratio of load $p_{t}$ that is powered at time $t$, to total load required $\left(h_{t}\right)$. The overall utility $u^{a}$ is the sum of these ratios, given by:

$$
u^{a}=\sum_{t=1}^{n} \frac{p_{t}}{h_{t}}
$$

Thus, the goal of an agent is to power as much load as possible to maximise its utility. The battery is useful here as it gives the agent flexibility in deciding when to store and when to use energy and thus, it enables the agent to find an optimal energy allocation, $\boldsymbol{p}^{*}$, to maximize utility, given by:

$$
\boldsymbol{p}^{*} \underset{p_{t} \in \boldsymbol{p}}{\operatorname{argmax}} \sum_{t=1}^{n}\left(\frac{p_{t}}{h_{t}}\right)
$$

This can be transformed to a linear programming model subjected to the following constraints:

Constraint 1: At any given time $\mathrm{t}$, the allocated power $p_{t} \in \boldsymbol{p}$ depends on the generated power $g_{t}$, battery charging flow $c_{t}$, discharging flow $d_{t}$ and link flow $l_{t}$.

$$
p_{t}=g_{t}-c_{t}+d_{t}+l_{t}
$$

Constraint 2: The current battery state $q_{t}$ depends on the last battery state $q_{(t-1)}$, charge $c_{(t-1)}$ and discharge $d_{(t-1)}$. The charge flow $c_{t} \in c$ is subjected to the battery efficiency $e$. Also, the first state of the battery $q_{1}$ must equal the last battery state of the battery $q_{n}$ to ensure there is no net change of battery charge over the day so that the utility remains dependent only on the energy generated in $n$ time periods.

$$
q_{t}= \begin{cases}q_{(t-1)}+e \times c_{(t-1)}-d_{(t-1)} & , t>1 \\ q_{n}+e \times c_{n}-d_{n} & , t=1\end{cases}
$$

Constraint 3: Allocated power $p_{t}$ must not exceed load $h_{t}$.

$$
p_{t} \leq h_{t} \quad \forall p_{t} \in \boldsymbol{p}, h_{t} \in \boldsymbol{h}
$$

Constraint 4: The battery state $q_{t}$ must not exceed the maximum capacity $s$. Also, the battery state cannot be negative, i.e. energy must be stored before it is drawn.

$$
0 \leq q_{t} \leq s \quad \forall q_{t} \in \boldsymbol{q}
$$

Constraint 5: At any time period $t$, the battery charge flow $c_{t}$ must not exceed the maximum charge limit $c_{\max }$. Also charge flow is always positive.

$$
0 \leq c_{t} \leq c_{\max } \quad \forall c_{t} \in \boldsymbol{c}
$$


Constraint 6: At any time period $t$, the battery discharge flow $d_{t}$ must not exceed the maximum discharge limit $d_{\max }$. Also discharge flow is always positive.

$$
0 \leq d_{t} \leq d_{\max } \quad \forall d_{t} \in \boldsymbol{d}
$$

Constraint 7: Wasted energy $w_{t}$ is always positive and cannot exceed the energy $k_{t}$ that can be generated at time $t$.

$$
0<w_{t}<k_{t} \quad \forall w_{t} \in \boldsymbol{w}, k_{t} \in \boldsymbol{k}
$$

Constraint 8: Battery efficiency must be between 0 to 1 (i.e. $0 \%$ to $100 \%)$.

$$
0 \leq e \leq 1
$$

Now, an agent can compute an energy allocation $\boldsymbol{p}^{*}$ which maximizes its utility via Equation 2 and constraints $\left\{c_{1}, \ldots, c_{8}\right\}$. When an agent's objective is to compute a link flow that can maximize its utility, it can compute it as the following. Given Equation 1, substituting for $p_{t}$ from $c_{1}$ :

$$
u^{a}=\sum_{t=1}^{n}\left(\frac{g_{t}-c_{t}+d_{t}+l_{t}}{h_{t}}\right)
$$

The objective is then to find the optimal link flow $l^{*}$, such that Equation 3 is maximized:

$$
\boldsymbol{l}^{*}=\underset{l_{t} \in \boldsymbol{l}}{\operatorname{argmax}} \sum_{t=1}^{n}\left(\frac{g_{t}-c_{t}+d_{t}+l_{t}}{h_{t}}\right)
$$

subjected to all constraints $\left\{c_{1}, \ldots, c_{8}\right\}$ as listed above.

Given this model, the problem of finding an energy exchange agreement between two self-interested agents can now be simplified to the problem of finding an agreed link flow, $\boldsymbol{l}$, that provides each agent with greater utility than when disconnected (i.e., when $l=0$ ). However, the set of all such links is exponential in the number of time periods and thus, it is a challenging task for both agents to agree on a link given that each agent will prefer a different one (i.e., the link which maximizes its own utility).

In the next section, we elaborate on a game theoretic solution to find an agreed link flow between agents. In so doing, we establish the benchmark against which we compare our protocol (which we detail in Section 4).

\section{The Nash Bargaining Solution: A Theoretical Benchmark}

Given the model above, we now describe a game theoretic solution, known as the Nash bargaining solution [Nash, 1950; 1953], which allows the agents to find a satisfying solution for both. Nash bargaining solution (NBS) is defined by four axioms that must hold true: (i) invariance to utility scales (it is independent of how agents scale their utility) (ii) symmetry (it does not depend on the identity of agents), (iii) Paretooptimal (the outcome is such that no agent can improve upon its utility without making another agent worse off) and independence from irrelevant alternatives. These axioms define a unique solution that maximizes the product of gains in utility of agents. Note that the NBS does not provide the mechanism by which agents might reach agreement, but it serves as the solution concept against which we will compare our own protocol. In the context of energy exchange, the gain in utility comes from the fact that via exchange an agent can avoid energy storage losses and utilize energy that will be unused otherwise. To be clearer on this point, if an agent has $100 \%$ efficient battery and infinite storage, it cannot increase its utility via exchange.

Finding the NBS in the energy exchange problem basically involves finding the link flow between agents that maximizes the product of gains in their utility. Assuming two agents $a$ and $b$, let $d^{a}$ and $d^{b}$ be the utilities that agents can avail when they are disconnected (also called disagreement utilities). These disagreement utilities are the maximum utilities that $a$ and $b$ can get when the link flow $\boldsymbol{l}=0$, as we explained in Section 2. Let the solution set $S_{N B S}$ be the set of all feasible link flows between $a$ and $b$. Nash [1953] requires this set to be compact ${ }^{1}$ and convex ${ }^{2}$ for the solution to be unique. Since agents will only exchange if they get more utility than their disagreement utilities, $\forall \boldsymbol{l} \in S_{N B S}:\left(u^{a}(\boldsymbol{l}), u^{b}(\boldsymbol{l})\right)>$ $\left(d^{a}, d^{b}\right)$. Given the set $S_{N B S}$, the Nash bargaining solution, $\boldsymbol{l}_{N B S}$, is obtained by:

$$
\boldsymbol{l}_{N B S}=\underset{\boldsymbol{l}}{\operatorname{argmax}}\left[u^{a}(\boldsymbol{l})-d^{a}\right] \times\left[u^{b}(\boldsymbol{l})-d^{b}\right]
$$

This equation is subjected to constraints listed in Section 2 for both agents $a$ and $b$. Given compactness and convexity, computing the optimal solution is straight forward using convex optimization. However, interdependency between issues gives rise to a non-convex solution set with multiple NBS [Fujita, Ito, and Klein, 2010] whereby stochastic optimization techniques (we use simulated annealing) is needed to find the NBS. In general, negotiation mechanisms which aim to reach the NBS come with the implicit assumption that agents reveal their information truthfully either to each other or to a mediator so that the NBS can be computed. In a society of honest agents, where time and computational power are abundant, such mechanisms can be a good candidate to solve the problem of energy exchange. However, these assumptions do not hold in our setting thus the direct use of NBS is not valid in our setting. However, it can serve as an upper bound of the performance of a negotiation protocol. In the next section, we propose a negotiation protocol which does not require such assumptions and can be used to reach energy exchange agreements in the settings describe earlier.

\section{Energy Exchange Protocol}

Here we propose our energy exchange protocol (EEP) which allows agents to make offers to each other in order to reach an agreement on the link flow between them. We call these offers link flow offers and the agreement, an exchange agreement. The protocol imposes restrictions on the offers that agents can make and dictates that whichever offer minimizes the load on the link is selected as the exchange agreement. The restrictions on the offers reduce the set of feasible solutions between agents and ensure that the solution is reached with certain properties (listed in Section 4.2).

Before we define our protocol, we define our assumptions and terminology. We consider each exchange agreement in isolation, independent of the past and future exchange agreements. Also, we consider exchange over finite time (e.g. a

\footnotetext{
${ }^{1} \mathrm{~A}$ set is compact if it is closed and bounded.

${ }^{2} \mathrm{~A}$ set $C$ is convex if for all $x$ and $y$ in $C$ and all $t$ in the interval $[0,1]$, the point $(1-t) x+t(y)$ is in $\mathrm{C}$.
} 
day) which can be divided into exchange periods. An exchange period is a unit of time for energy exchange and consists of at least one time period. A time period is an atomic unit of time and each entry in a profile (e.g. energy generation) is described against a single time period. Given this protocol, we assume two agents $a$ and $b$, with utility functions $u^{a}$ and $u^{b}$, disagreement utilities $d^{a}$ and $d^{b}$ respectively, and let $S_{p}$ be the set of possible link flows between them, i.e. $\forall \boldsymbol{l} \in S_{p}:\left(u^{a}(\boldsymbol{l}), u^{b}(\boldsymbol{l})\right) \geq\left(d^{a}, d^{b}\right)$, the same set as defined in Section 3. The EEP imposes three restrictions, $r_{1}, r_{2}$ and $r_{3}$ on the offers and allows agents to make offers at most once such that the negotiation results in a dominant strategy equilibrium and a Pareto-optimal outcome. The outcome (the agreed link flow) is denoted by $\boldsymbol{l}^{\boldsymbol{e x}}$. Figure 1 describes the EEP in detail.

\subsection{Computing the Valid Link Flow Offers}

Given the protocol, we now define how agents can compute valid link flow offers. As discussed in Section 2, each agent will prefer the link flow that maximizes its own utility. An agent making an offer can compute a valid link flow offer which maximizes its utility by using Equation 4 with two more constraints, $\left\{r_{1}, r_{2}\right\}$ (in addition of $\left(\left\{c_{1}, \ldots c_{8}\right\}\right)$, and an agent making a counter-offer needs to include a further constraint $r_{3}$. In fact, agents can offer any link flow provided it is valid, not just the one that maximizes its utility. However, in Section 4.2, we prove that the dominant strategy for agents is to offer the link flow that maximizes its utility, leaving no need for them to strategize.

Before we explain the properties of the EEP, we give an intuitive example to show how it will work in action:

Example 1. Imagine that in a society of agents, the following are the already agreed conventions:

1. The total time of an exchange is 24 hours. The exchange starts at 0600 hours local time and ends at 0600 hours next day.

2. This day is divided into two exchange periods, each consists of 6 two-hours-long time periods.

Given these conventions, an agent a computes a valid link flow offer, $l^{\boldsymbol{a}}=(2,2,2,2,2,2,-2,-2,-2,-2,-2,-2)$, which maximizes its utility. Agent a makes this offer to agent $b$. However, Agent $b$ finds that its utility is maximized with the link flow, $\boldsymbol{l}^{\boldsymbol{b}}=(1,1,1,1,1,1,-1,-1,-1,-1,-1,-1)$. Since $\boldsymbol{l}^{b}<^{3} \boldsymbol{l}^{a}, b$ makes this offer to a. Agent a accepts this offer and exchange takes place as per $\boldsymbol{l}^{b}$.

\subsection{Properties of Our protocol}

Before outlining the properties, we describe our notation. Let $S$ be the set of valid link flows between $a$ and $b$ obeying the rules defined by the EEP. If $\exists \boldsymbol{l} \in S: u^{a}(\boldsymbol{l})>0$ then $\forall \boldsymbol{l}^{\prime} \in S: 0<\boldsymbol{l}^{\prime}<\boldsymbol{l}, u^{a}\left(\boldsymbol{l}^{\prime}\right)>0$. For example, if agent $a$ prefers an exchange $(2,2,-2,-2)$, then $(1,1,-1,-1)$ is also a feasible link flow for $a$, since $(1,1,-1,-1) \in S$ and $(1,1,-1,-1)<(2,2,-2,-2)$, although $u^{a}[(1,1,-1,-1)]<u^{a}[(2,2,-2-2)]$. It actually follows that given a link $\boldsymbol{l}^{*} \in S: u^{a}\left(\boldsymbol{l}^{*}\right)>u^{a}(\boldsymbol{l}) \forall \boldsymbol{l} \in$ $S: \boldsymbol{l} \neq \boldsymbol{l}^{*}$, then all $\boldsymbol{l} \in S: 0<\boldsymbol{l}<\boldsymbol{l}^{*}$ are feasible and $u^{a}$ is a strictly monotonically increasing function on this range.

\footnotetext{
${ }^{3}$ The inequality here is the vector inequality.
}

\section{Energy Exchange Protocol (EEP)}

1. Agent $a$ submits a valid link flow offer $\boldsymbol{l}^{a}$ to agent $b$. An offer is valid if it meets the following criteria.

- The offer must have exactly two exchange periods. Each exchange comprises of an equal number of consecutive time periods. The sum of magnitude of energy in time periods of an exchange period must be equal to other exchange period.

$$
\boldsymbol{l}=\left(l_{1}, \ldots . . l_{n}\right) \in S:\left|\sum_{t=1}^{n / 2} l_{t}\right|=\left|\sum_{t=n / 2+1}^{n} l_{t}\right|
$$

- The amount of energy in each time period is equal.

$$
\boldsymbol{l}=\left(l_{1}, \ldots . . l_{n}\right) \in S: \forall l_{t} \in \boldsymbol{l}:\left|l_{t}\right|=\left|l_{t+1}\right|
$$

2. On receiving the offer $\boldsymbol{l}^{a}$, Agent $b$ has three options:

- Reject: $b$ can reject an offer, e.g. when exchange is not beneficial to $b$. It happens when $\neg \exists \boldsymbol{l} \in S: u^{b}(\boldsymbol{l})>0$. Agent $b$ indicates its rejection by sending the REJECT message. The agreed link flow is $\boldsymbol{l}^{\boldsymbol{e x}}=0$. The EEP terminates.

- Accept: $b$ can accept $l^{a}$ by sending the ACCEPT message. The agreed link flow is $\boldsymbol{l}^{\boldsymbol{e x}}=\boldsymbol{l}^{a}$. The EEP terminates.

- Counter-offer: Finally, $b$ can make a counter offer $\boldsymbol{l}^{\boldsymbol{b}}$ which meets above criteria $\left(r_{1}, r_{2}\right)$. In addition, it must ensure that:

$$
\boldsymbol{l}^{b}<\boldsymbol{l}^{a}
$$

3. On receiving the counter offer, $\boldsymbol{l}^{b}$, Agent $a$ has two options:

- Accept: It can accept $\boldsymbol{l}^{b}$ and send the ACCEPT message to $b$. The agreed link flow is $l^{e x}=l^{b}$. The EEP terminates.

- Reject: It can reject $\boldsymbol{l}^{\boldsymbol{b}}$ and send the REJECT message to $b$. The link flow is not agreed, $\boldsymbol{l}^{\boldsymbol{e x}}=0$. The EEP terminates.

\section{Figure 1: The Negotiation Protocol}

This monotonicity arises from the fact that the negotiation has been reduced to a single-issue, i.e. the amount of flow in one time period (since the amount of energy in each time period must be equal - see $r_{2}$ ). Now we prove the properties.

\section{Dominant Strategy Equilibrium}

Theorem 1. The agent making an initial offer has a dominant strategy which is to offer the link that maximizes its own utility.

Proof. Let's assume two agents $a$ and $b$, and $\boldsymbol{l}^{a} \in S$ is the link that optimizes the utility of agent $a$. Let's imagine that $a$ wants to manipulate the protocol by reporting some other link $\hat{\boldsymbol{l}}^{a} \in S$ to agent $b$. Table 1 lists all possible scenarios that $a$ faces in this case. It is evident that reporting $\boldsymbol{l}^{a}$ weakly dominates reporting $\hat{l}^{a}$. Specifically, in the first two cases, reporting $\hat{l}^{a}$ gives the same utility as reporting $\boldsymbol{l}^{a}$, however it is strongly dominated in the rest of the four cases (unless $\hat{l}^{a}=l^{a}$ ). Thus, an agent maximizes its utility by reporting $\hat{l}^{a}=\boldsymbol{l}^{a}$.

Theorem 2. An agent making a counter-offer has a dominant strategy to offer the link that maximizes its own 


\begin{tabular}{|c|c|c|c|c|c|}
\hline \multirow[t]{2}{*}{ Case } & \multicolumn{2}{|c|}{ Agent $a$ reports $\hat{l}^{a}$} & \multicolumn{2}{|c|}{ Agent $a$ reports $l^{a}$} & \multirow[t]{2}{*}{ Result } \\
\hline & $\begin{array}{l}\text { Chosen Link } \\
\min \left(\hat{\boldsymbol{l}}^{a}, \boldsymbol{l}^{\boldsymbol{b}}\right)\end{array}$ & $u^{a}$ & $\begin{array}{l}\text { Chosen Link } \\
\min \left(\boldsymbol{l}^{\boldsymbol{a}}, \boldsymbol{l}^{\boldsymbol{b}}\right)\end{array}$ & $u^{a}$ & \\
\hline$\hat{\boldsymbol{l}}^{a} \geq \boldsymbol{l}^{a} \geq \boldsymbol{l}^{b}$ & $l^{b}$ & $u^{a}\left(l^{b}\right)$ & $l^{b}$ & $u^{a}\left(l^{b}\right)$ & $u^{a}\left(\boldsymbol{l}^{b}\right)=u^{a}\left(\boldsymbol{l}^{b}\right)$. \\
\hline $\boldsymbol{l}^{a} \geq \hat{\boldsymbol{l}}^{a} \geq \boldsymbol{l}^{b}$ & $l^{b}$ & $u^{a}\left(l^{b}\right)$ & $l^{b}$ & $u^{a}\left(l^{b}\right)$ & $u^{a}\left(\boldsymbol{l}^{b}\right)=u^{a}\left(\boldsymbol{l}^{b}\right)$ \\
\hline $\boldsymbol{l}^{a} \geq \boldsymbol{l}^{b} \geq \hat{\boldsymbol{l}}^{a}$ & $\hat{\boldsymbol{l}}^{a}$ & $u^{a}\left(\hat{\boldsymbol{l}}^{a}\right)$ & $l^{b}$ & $u^{a}\left(l^{b}\right)$ & $u^{a}\left(\hat{\boldsymbol{l}}^{a}\right) \leq u^{a}\left(\boldsymbol{l}^{b}\right) \because \hat{\boldsymbol{l}}^{a} \leq \boldsymbol{l}^{b} \leq \boldsymbol{l}^{a}$ (monotonicity) \\
\hline $\boldsymbol{l}^{b} \geq \boldsymbol{l}^{a} \geq \hat{\boldsymbol{l}}^{a}$ & $\hat{\boldsymbol{l}}^{a}$ & $u^{a}\left(\hat{\boldsymbol{l}}^{a}\right)$ & $l^{a}$ & $u^{a}\left(l^{a}\right)$ & $u^{a}\left(\hat{\boldsymbol{l}}^{a}\right) \leq u^{a}\left(\boldsymbol{l}^{\boldsymbol{a}}\right) \because u^{a}\left(\boldsymbol{l}^{a}\right)>u^{a}(\boldsymbol{l}) \forall \boldsymbol{l} \in S: \boldsymbol{l} \neq \boldsymbol{l}^{\boldsymbol{a}}$ \\
\hline$l^{b} \geq \hat{l}^{a} \geq l^{a}$ & $\hat{\boldsymbol{l}}^{a}$ & $u^{a}\left(\hat{\boldsymbol{l}}^{a}\right)$ & $l^{a}$ & $u^{a}\left(l^{a}\right)$ & $u^{a}\left(\hat{\boldsymbol{l}}^{a}\right) \leq u^{a}\left(\boldsymbol{l}^{\boldsymbol{a}}\right) \because u^{a}\left(\boldsymbol{l}^{\boldsymbol{a}}\right)>u^{a}(\boldsymbol{l}) \forall \boldsymbol{l} \in S: \boldsymbol{l} \neq \boldsymbol{l}^{\boldsymbol{a}}$ \\
\hline$\hat{l}^{a} \geq \boldsymbol{l}^{b} \geq \boldsymbol{l}^{a}$ & $l^{b}$ & $u^{a}\left(\boldsymbol{l}^{b}\right)$ & $l^{a}$ & $u^{a}\left(l^{a}\right)$ & $u^{a}\left(\hat{\boldsymbol{l}^{\boldsymbol{b}}}\right) \leq u^{a}\left(\boldsymbol{l}^{\boldsymbol{a}}\right) \because u^{a}\left(\boldsymbol{l}^{\boldsymbol{a}}\right)>u^{a}(\boldsymbol{l}) \forall \boldsymbol{l} \in S: \boldsymbol{l} \neq \boldsymbol{l}^{\boldsymbol{a}}$ \\
\hline
\end{tabular}

Table 1: Reporting the link, $l^{a}$, which maximizes its utility, is the dominant strategy for agent $a$.

utility.

Proof. Imagine agent $a$ with $\boldsymbol{l}^{a} \in S$ which optimizes its utility and $\boldsymbol{l}^{b} \in S$, the offer it has received from agent $b$. If $\hat{l}^{a}$ is the link that $a$ wants to report $b$ to manipulate the protocol, then Table 1 lists all the possible scenarios. Again, reporting $\boldsymbol{l}^{a}$ weakly dominates $\hat{\boldsymbol{l}}^{a}$.

It is evident that revealing the link that maximizes its utility, is a weakly dominant strategy for both initialoffer and counter-offer making agents. As a by-product, this property also renders the order, in which agents make offers to each other, irrelevant. The solution is the same no matter which agent commences the negotiation. The result is always a dominant strategy equilibrium.

\section{Pareto-Optimality}

Let's imagine that $\exists \boldsymbol{l} \in S: u^{a}(\boldsymbol{l})>0$ and $u^{b}(\boldsymbol{l})>0$. As proved above, agent $a$ and $b$ will reveal truthfully the links $\boldsymbol{l}^{a}$ and $\boldsymbol{l}^{b}$, that maximizes their utilities $u^{a}$ and $u^{b}$. Assume that $\boldsymbol{l}^{a}>\boldsymbol{l}^{b}$, then the EEP dictates that $\boldsymbol{l}^{b}$ is the agreed link flow. Since $\boldsymbol{l}^{b}$ maximizes $u^{b}$ and it is unique $\left(u^{b}\right.$ is strictly monotonic), any other link flow which increases $u^{a}$ will decrease $u^{b}$, hence the protocol is Pareto-optimal.

\section{Tractability}

Our solution is tractable as each agent needs to make at most one offer. Also, an optimal offer can be computed using standard linear programming solver (Section 2).

\section{Empirical Evaluation}

Having proven the properties of our protocol, we now set-up a realistic example to demonstrate its practical applicability. To this end, we consider an example of energy exchange between two agents, where one agent has a $1.5 \mathrm{~kW}$ wind turbine and the other has a $1.75 \mathrm{~kW}$ solar panel. The energy generation data for the wind turbine comes from a wind farm near Lugo, Northwest Spain (www.sotaventogalicia.com), while the output of the solar panel is estimated to be directly proportional to the daily radiance for the same region (www.re.jrc.ec.europa.eu/pvgis/apps/radday.php). We use data for July 2010, estimate the average generation for a day and scale it to match the output of a $1.5 \mathrm{~kW}$ wind turbine and a $1.75 \mathrm{~kW}$ solar panel. At present, the load requirements of homes in remote areas are not available so we use load data, recorded and provided by a UK electric company in lowincome homes equipped with smart meters. We also assume that agents have identical batteries that have a maximum capacity, $s=20 \mathrm{kWh}$, a maximum charging rate, $c=4 \mathrm{kWh}$, a maximum discharging rate, $d=-4 \mathrm{kWh}$ and efficiency, $e=90 \%$. We note that the generation data for agents have some disparity and all other data (including loads) and batteries are identical for both agents. Given these profiles, agents can compute their utilities (without exchange) using a linear programming solver (see Section 2). We use IBM ILOG CPLEX, a powerful optimizer, which provides easy and rich methods to model constraints, variables and objective functions and flexible interfaces to many development tools.

For a comparative analysis of our protocol, we compute the utilities that agents can get (i) without exchange, (ii) by computing the NBS using simulated annealing (see Section 3 ) and (iii) by using our EEP (Section 4.1). Moreover, we consider how these utilities vary as we reduce the battery flow and storage capacity of agent $a$ as shown in Figure 2.

Consider first Figure 2(a) which shows the utilities of agent $a$ and $b$, and the total energy saved via exchange, as the battery efficiency of agent $a$ is reduced. All the other factors, (including the battery efficiency of agent $b$ ) are kept constant. We can see that agents can increase their utilities via exchange. The amount by which it increases depends on the type of solution used and the battery efficiency. The total energy saved via exchange is the energy which would otherwise be lost (either in storage loss or due to limited battery flow or storage - Section 3) without exchange. We also see that as the battery efficiency of agent $a$ is reduced, so does its ability to meet its load, therefore, its utility reduces. While for $b$, this reduction in the battery efficiency of $a$ does not affect its utility for without exchange and with the EEP, however, for the NBS its utility increases. The reason that $b$ gets better utility via the NBS as the battery efficiency of $a$ is reduced, is because storage loss for $a$ increases and thus, the amount of energy that can be saved in exchange via exchange increases, as shown in Figure 2(a). However, this saved energy is the result of cooperation between agents and the NBS divides it between both agents and hence, the increase in utility of $b$. Thus, with reduction in the battery efficiency, the energy exchange becomes more beneficial as agents can reduce their storage losses by exchanging energy.

We also note that the NBS gives both agents better utilities compared to the EEP, however, its computation comes with the assumptions discussed in Section 3. To compare the EEP against the NBS, we measure the difference in the energy saved using these solutions. We observe that this difference depends on the battery efficiency of $a$ and that the EEP can save from $29 \%$ to $84 \%$ (Figure 2(a) and Figure 2(b), respectively) compared to the energy saved with the NBS.

Figure 2(b) shows the utilities of the agents as the battery capacity of agent $a$ is reduced. Again, as in Figure 2(a), agents can get better utilities with exchange. Also evident is the fact that as storage is reduced, energy exchange becomes 

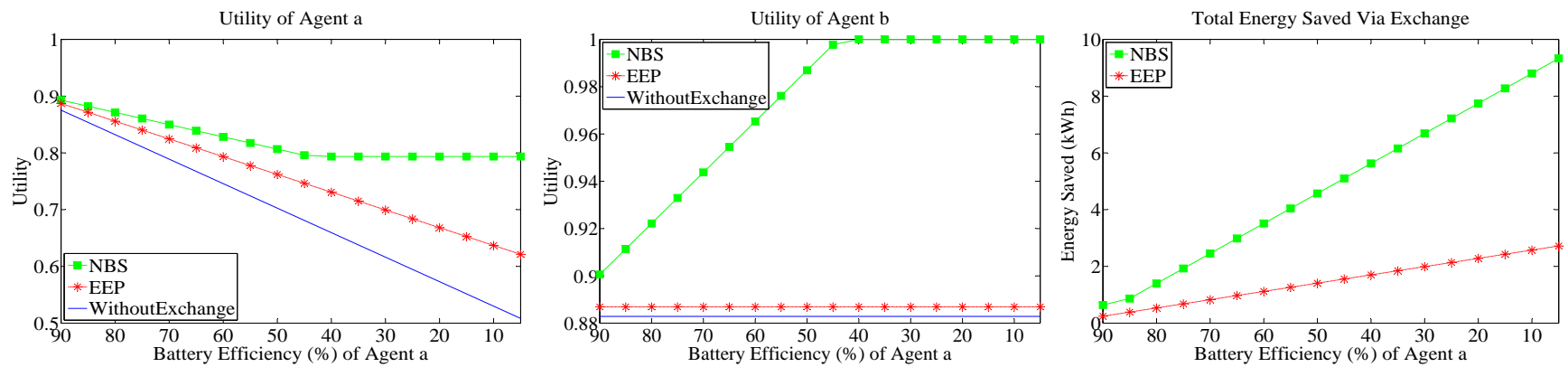

(a) Utility of agents and total energy saved verses battery efficiency of agent $a$
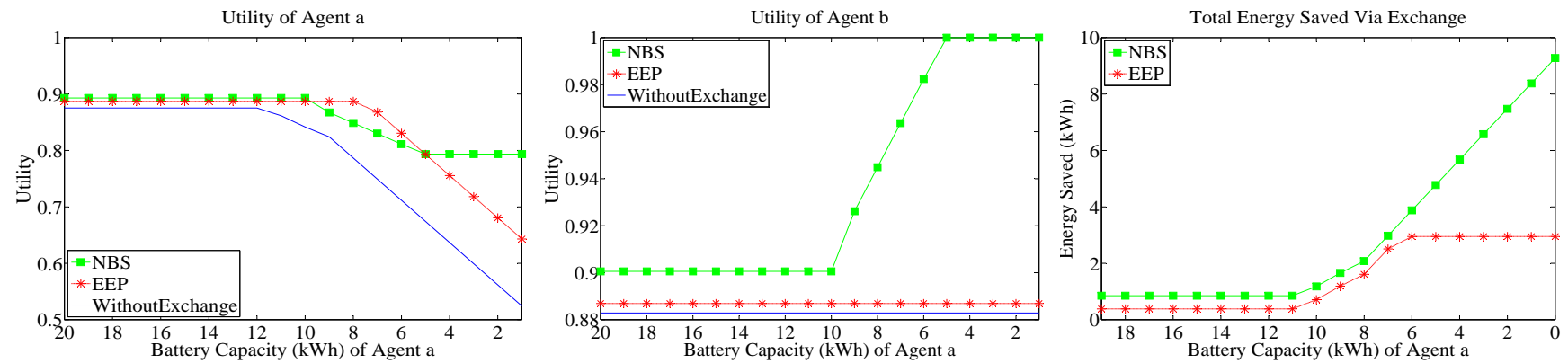

(b) Utility of agents and total energy saved versus battery capacity of agent $a$

Figure 2: Total energy saved and utility of agents (without exchange, with EEP and with NBS), as battery efficiency and capacity is reduced.

more useful as agents can exchange energy instead of storing it. An important observation here is that an agent can maintain the same level of utility with a smaller battery via exchange that it obtains otherwise. For example, we see that $a$ maintains the same level of utility in exchange via our protocol with a $7 \mathrm{kWh}$ battery that it gets without exchange with an $11 \mathrm{kWh}$ battery. This corresponds to almost a $37 \%$ reduction in battery size. Though, the same phenomenon can be seen for battery efficiency, what makes battery capacity more interesting here is the strongly correlated relationship between the battery capacity and cost, and thus, reducing the battery size will significantly reduce its cost.

\section{Conclusion and Future Work}

The problem of energy exchange in homes is an interdependent multi-issue negotiation problem. Such problems are complex and computationally expensive to negotiate over. We present a novel negotiation protocol, the EEP, which imposes certain restrictions to reduce the complexity of the energy exchange problem and leads to a dominant strategy equilibrium and a Pareto-optimal outcome. We prove these properties of the EEP and demonstrate via example that agents can increase their utility (up to $22 \%$ ), reduce the required storage capacity (up to $37 \%$ less) and that the energy saved using the EEP can be up to $84 \%$ of the energy that can be saved using the NBS. In our example, only the energy generation data for agents is different and introducing diversity in other data (e.g. loads) can make energy exchange more useful and lead to even better results. We conclude from our presented work and the empirical results that energy exchange between homes has the obvious potential of better energy management. Although, we have confined our vision to homes in developing countries, energy exchange can also be useful in the smart grid vision. In future work, we aim to extend this model and investigate a similar set of rules to reach a dominant strategy equilibrium in multiagent society with less re- strictions. We also aim to investigate the cases where energy generation is uncertain and where loads are deferrable.

\section{References}

[Ehtamo et al., 1988] Ehtamo, H.; Ruusunen, J.; Kaitala, V.; and Hamalainen, R. 1988. Solution for a dynamic bargaining problem with an application to resource management. Jr. of Optimization Theory 59(3):391-405.

[Fujita, Ito, and Klein, 2010] Fujita, K.; Ito, T.; and Klein, M. 2010. A secure and fair protocol that addresses weaknesses of the Nash bargaining solution in nonlinear negotiation. Group Decision and Negotiation. 1-19.

[Hattori, Klein, and Ito, 2007] Hattori, H.; Klein, M.; and Ito, T. 2007. A multi-phase protocol for negotiation with interdependent issues. In Proc. of IAT, 153 -159, Sydney, Australia.

[Hindriks, Jonker, and Tykhonov, 2006] Hindriks, K.; Jonker, C.; and Tykhonov, D. 2006. Eliminating interdependencies between issues for multi-issue negotiation. Cooperative Info. Agents, Lecture Notes in Computer Science. Springer.

[IEA, 2008] International Energy Agency. World Energy Outlook.

[Ito, Hattori, and Klein, 2007] Ito, T.; Hattori, H.; and Klein, M. 2007. Multi-issue negotiation protocol for agents: exploring nonlinear utility spaces. In Proc. of IJCAI, Hyderabad, India

[Nash, 1950] Nash, J. 1950. The bargaining problem. Econometrica $18(2): 155$.

[Nash, 1953] Nash, J. 1953. Two-person cooperative games. Econometrica 21(1):128-140.

[Rosenschein and Zlotkin, 1994] Rosenschein, J. S., and Zlotkin, G. 1994. Rules of encounter: designing conventions for automated negotiation among computers. Cambridge, MA, USA: MIT Press.

[Ruusunen, Ehtamo, and Hamalainen, 1991] Ruusunen, J.; Ehtamo, H.; and Hamalainen, R. 1991. Dynamic cooperative electricity exchange in a power pool. Systems, Man and Cybernetics, IEEE Transactions on 21(4):758-766. 